\title{
X-Ray Reflectivity Studies of a Microemulsion Surface
}

\section{Citation}

Schwartz, D. K., A. Braslau, B. Ocko, Peter S. Pershan, J. Als-Nielsen, and J. S. Huang. 1988. Xray reflectivity studies of a microemulsion surface. Physical Review A 38(11): 5817-5824.

\section{Published Version}

doi:10.1103/PhysRevA.38.5817

\section{Permanent link}

http://nrs.harvard.edu/urn-3:HUL.InstRepos:8609121

\section{Terms of Use}

This article was downloaded from Harvard University's DASH repository, and is made available under the terms and conditions applicable to Other Posted Material, as set forth at http:// nrs.harvard.edu/urn-3:HUL.InstRepos:dash.current.terms-of-use\#LAA

\section{Share Your Story}

The Harvard community has made this article openly available.

Please share how this access benefits you. Submit a story.

Accessibility 


\title{
$\mathrm{X}$-ray reflectivity studies of a microemulsion surface
}

\author{
D. K. Schwartz, A. Braslau, ${ }^{*}$ B. Ocko, ${ }^{\dagger}$ and P. S. Pershan \\ Division of Applied Physics and Department of Physics, Harvard University, Cambridge, Massachusetts 02138 \\ J. Als-Nielsen \\ Risö National Laboratory, DK-4000 Roskilde, Denmark \\ J. S. Huang \\ Corporate Research Science Laboratories, Exxon Research and Engineering Company, Annandale, New Jersey 08801
}

(Received 28 March 1988)

\begin{abstract}
The surface structure of the AOT (sodium di-2-ethylsulfosuccinate) microemulsion system with equal volume fractions of $\mathrm{D}_{2} \mathrm{O}$ and decane, for AOT volume fractions of $0.42,0.305$, and 0.181 , has been studied by a combination of $x$-ray specular reflectivity and scattering from the bulk. Scattering from the bulk material below the surface is consistent with Kotlarchyk's interpretation of densely packed spherical micelles. Specular reflectivity from the surface implies a surface electron-density profile consistent with one to two layers of microemulsion droplets. The size of and distance between droplets is consistent with the respective values for the droplets in the bulk phase.
\end{abstract}

\section{INTRODUCTION}

In the presence of suitable concentrations of a surfactant, mixtures of oil and water can form a single homogeneous phase consisting of a clear isotropic solution of microscopic micelles whose size (i.e., generally in the range of 50 to $1000 \AA$ ) is determined by a combination of entropy and energy considerations. ${ }^{1}$ An essential feature is the formation of a monolayer of amphiphilic surfactant molecules at the surface separating the oil and water. Under the usual conditions, the surface tension, or free energy per unit area, of this interface is positive and the system optimizes the micelle size at some expense in interfacial energy. The addition of a suitably chosen fourth component called a cosurfactant, usually an alcohol, allows one to vary both the surface tension and the interfacial curvature so as to obtain a variety of other phases, e.g., lamella, hexagonal, etc. ${ }^{2}$ In particular, there are conditions for which the internal surfaces of the resultant phases have radii of curvatures as small as $20 \AA$. These are generally referred to as microemulsions. Recent theoretical studies explain the relative stability of some of these phases in terms of competition between the bending energy of the surfactant film and the packing energy of the hydrocarbon tails of the surfactant. ${ }^{3,4}$

Microemulsions formed from mixtures of AOT (sodium di-2-ethylsulfosuccinate), water, and oil (without a cosurfactant) are one example of a system that has been extensively studied as a model for the more general microemulsion. ${ }^{2,5-10}$ The AOT molecule is amphiphilic with two bulky branched hydrocarbon chains. If the surfactant molecules are closely packed to form a flat interface the bulky hydrophobic tails would interfere with one another. Since the system is not constrained to a flat interface the free energy can be lowered by allowing the film to bend spontaneously in order to give the tails more room. It follows that the AOT layer has a preferred ra- dius of curvature and under appropriate conditions this solution forms inverse micelles in which a water core is coated by a shell of AOT with the tails sticking out into the oil. In AOT microemulsion systems this kind of aggregate exists not only in dilute water mixtures but even with equal concentrations of water and oil., 911

Small angle neutron diffraction studies of bulk AOT microemulsions, containing equal volumes of heavy water $\left(\mathrm{D}_{2} \mathrm{O}\right)$ and decane, are consistent with a model of spherical micelles in which all of the AOT molecules in the sample are densely packed on the micelle surface. ${ }^{10}$ The observed structure factor is well represented by a model in which the local packing of the micelles is either bcc or fcc, with a thin layer of decane separating adjacent droplets. ${ }^{11}$ The study described here was undertaken in an attempt to determine how the local structure changes in the vicinity of the free surface between the microemulsion and air. In particular, the possibility that the flat microemulsion/air interface would force the surfactant film to be planar, thereby inducing a surface lamellar phase similar to the lamellar order observed near the surface of the isotropic phase of a liquid crystal is excluded by the present data. ${ }^{12}$ In contrast we will demonstrate below that the measurements prove that the dense packing of spherical micelles extends up to the microemulsion-vapor interface.

\section{BACKGROUND}

Previous experiments have demonstrated that $\mathrm{x}$-ray reflectivity can be used to study the structure of surfaces. $^{13-15}$ In particular, if $\langle\partial \rho / \partial z\rangle$ is the average derivative, describing the $z$ dependence of the electron density $\rho$ along the surface normal, the ratio of the measured reflectivity $R\left(Q_{z}\right)$ to the theoretical Fresnel reflectivity $R_{F}\left(Q_{z}\right)$ from a sharp, flat interface with no structure is 


$$
\frac{R\left(Q_{z}\right)}{R_{F}\left(Q_{z}\right)}=\left|\frac{1}{\rho^{\infty}} \int d z\langle\partial \rho / \partial z\rangle \exp \left(i Q_{z} z\right)\right|^{2},
$$

where $\rho^{\infty}$ is the electron density far below the surface,

$$
Q_{z}=\left|\mathbf{k}_{\text {out }}-\mathbf{k}_{\text {in }}\right|=(4 \pi / \lambda) \sin (\theta)
$$

and $\theta$ is measured with respect to the surface, i.e., $\theta=90^{\circ}$ is normal incidence. We have measured the $x$-ray reflectivity $R\left(Q_{z}\right)$ for three samples with equal volumes of $\mathrm{D}_{2} \mathrm{O}$ and decane and with volume fractions of AOT: (a) $\phi=0.420$, (b) 0.305 , and (c) 0.181 , that are identical to those used in the neutron diffraction experiments. ${ }^{11}$

The experiment was performed at the synchrotron at HASYLAB in Hamburg, West Germany. The spectrometer details have been published elsewhere; ${ }^{16}$ however, the basic idea is the following [Fig. 1(a)]: by tilting a germanium (111) monochromator downward by an angle $\tau$,
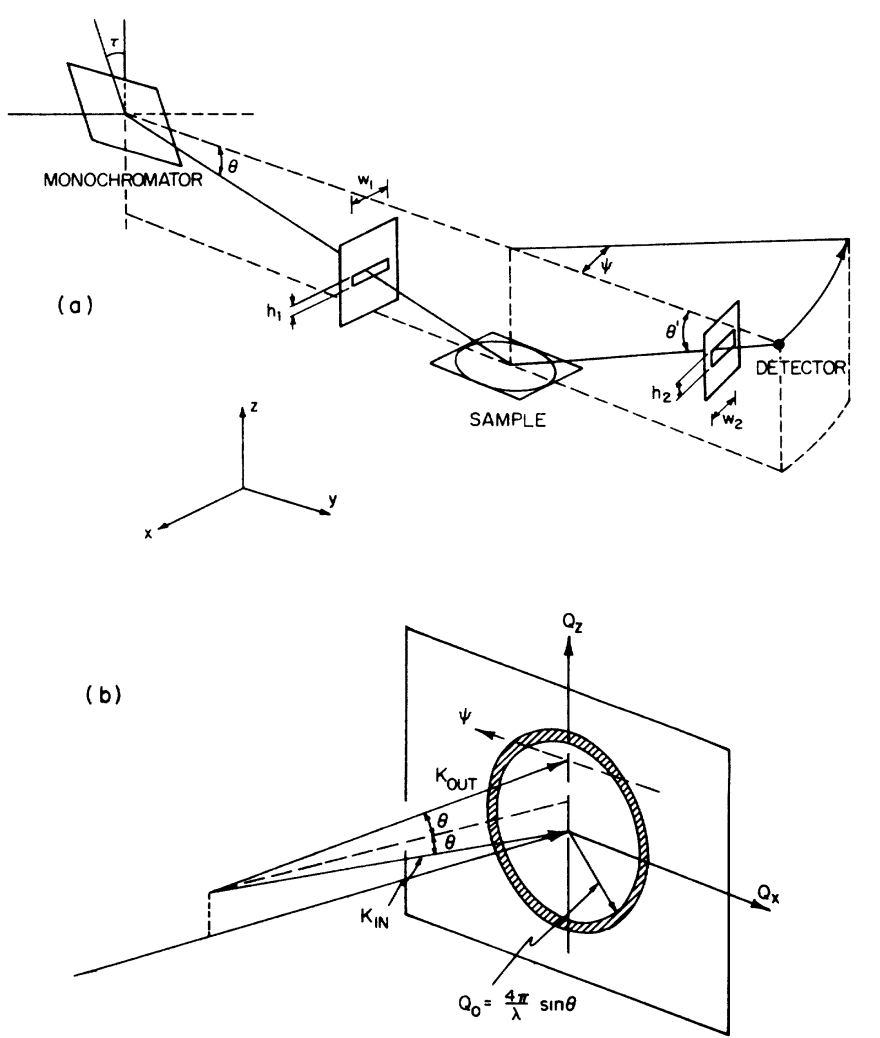

FIG. 1. (a) Schematic illustration of the spectrometer. The tilted monochromator crystal deflects the incident beam down by an angle $\theta$ from the horizontal as described in the text. The first slit trims the beam such that the beam projection does not overextend the sample. The combination of the slit before the sample and the slit before the detector sets the resolution. The detector can be positioned such that it detects $\mathrm{x}$ rays at any outgoing angle $\theta^{\prime}$ from the horizontal and angle out of the scattering plane $\psi$. (b) The scattered radiation as a function of $\mathbf{Q}=\mathbf{k}_{\text {out }}-\mathbf{k}_{\text {in }}$. The specular reflection is along the $Q_{z}$ axis while the scattering from the bulk forms a diffuse sphere with radius $Q_{0}$. The intersection of this sphere with the Ewald sphere forms the indicated ring of diffuse scattering. about horizontal axis normal to reciprocal lattice vector, the wavelength $\lambda=1.529 \AA$ selected from the incident white beam is deflected downward by an angle $\theta$. If $G$ is the magnitude of the $\mathrm{Ge}(111)$ reciprocal-lattice vector $(2 \pi / \lambda) \sin (\theta)=G \sin (\tau)$. The vertical opening $h_{1}$ of the input slit is chosen such that its length, as projected on the horizontal sample surface $h_{1} / \sin (\theta)$, is smaller than the length of the sample ( $\sim 57 \mathrm{~mm}$; however, because the sample top was not flat the effective size was closer to 30 $\mathrm{mm}$ ). Typical values of $h_{1}$ are of the order of $0.1 \mathrm{~mm}$. The width $w_{1}$, important for the resolution of the spectrometer in the $x$ direction and for the suppression of background, is of the order of 1 to $2 \mathrm{~mm}$. The distances from the monochromator to the sample and from the sample to the detector slit are $660 \mathrm{~mm}$. The wave vector for the measured radiation is determined by the location of the detector through the angles $\theta^{\prime}$ and $\psi$. The specular reflectivity measurements are made with $\theta^{\prime}=\theta$ and $\psi=0$. The width of the detector slit $w_{2}$ is set to be slightly larger than $w_{1}$ such that if the detector angle $\psi$ is varied there is a small range of angles for which the intensity of the signal is independent of $\psi$. Similarly the height $h_{2}$ is chosen such that there is a range of $\theta^{\prime}$, about the specular condition, for which the intensity is also fixed. In this way we are certain that the detector intercepts all of the specularly reflected signal. Comparison of the width of the curve of reflected intensity versus $\theta^{\prime}$ at $\psi=0$ with the width of a similar scan for the direct beam determines the macroscopic flatness of the surface. Typically the spread in outgoing angles $\Delta \theta^{\prime}$ is $\sim 0.05^{\circ}$.

If the detector arm is tuned off the specular direction by rotation by an angle $\psi$, the normal component of the wave-vector transfer remains fixed at $Q_{z}=(4 \pi / \lambda) \sin (\theta)$; however, the component perpendicular to $\hat{\mathbf{z}}$,

$$
\mathbf{Q}_{1} \equiv\left(Q_{x}^{2}+Q_{y}^{2}\right)^{1 / 2}=(4 \pi / \lambda) \cos (\theta) \sin (\psi / 2) \text {. }
$$

The detected intensity in this position is proportional to the differential cross section due to scattering from the bulk of the material below the surface

$$
\frac{d \sigma}{d \Omega} \propto \frac{r_{e}^{2}}{2 \mu} \int d^{3} r\langle\rho(\mathbf{r}) \rho(0)\rangle \exp \left(i \mathbf{Q}^{\prime} \cdot \mathbf{r}\right),
$$

where $\mathbf{Q}^{\prime}$ is the wave-vector transfer inside the material, $\mu$ is the absorption length for the $\mathrm{x}$-rays of wavelength $\lambda$ (i.e., $\mu \approx 0.1 \mathrm{~cm}$ ), and $r_{e}$ is the classical radius of the electron. We have neglected the Lorentz polarization corrections since the scattering angles are small. Although the depth of penetration, along $z$, of the $\mathrm{x}$ rays is of the order of $\mu^{-1} \sin (\theta)$ this angular factor is canceled by a projected area of the beam on the sample $\left(w_{1} \times h_{1}\right) / \sin (\theta)$. In addition, although $\mathbf{Q}_{1}$ is the same inside the material as in the vacuum, for small angles of incidence, $Q_{z}^{\prime}=\left(Q_{z}^{2}-Q_{c}^{2}\right)^{1 / 2}$ where $Q_{c}=(4 \pi / \lambda) \sin \left(\theta_{c}\right)$ and $\sin ^{2}\left(\theta_{c}\right)=\rho r_{e} \lambda^{2} / \pi$. For an unoriented powder of either fcc or bcc microcrystals of micelles with nearest-neighbor distance $d$, the first peak in the powder pattern at $Q_{0}$ would be conically distributed around the incident beam, as illustrated schematically in Fig. 1(b) at a cone angle of $\theta_{0}, Q_{0}=(4 \pi / \lambda) \sin \left(\theta_{0}\right)$ where $Q_{0}=\pi \sqrt{6} / d$. The diffuse scattering was measured for the same three samples as 
mentioned above, by scanning across the specular condition at a number of different fixed values of $Q_{z}$. The reciprocal space path for this scan is illustrated by the dashed line in Fig. 1(b).

\section{EXPERIMENTAL RESULTS}

Figure 2 displays the results, after subtracting diffuse background scattering, for the specular $x$-ray reflectivity $R\left(Q_{z}\right)$ for the three samples of AOT with volume fractions: (a) $\phi=0.420$, (b) 0.305 , and (c) 0.181 mentioned above. The diffuse background scattering was measured by tuning the spectrometer off of the specular condition in the $\psi$ direction by an amount several times the resolution. Figure 3 displays the same data divided by the theoretical form for the Fresnel reflectivity, $R_{F}\left(Q_{z}\right)$, as convolved with a Gaussian resolution function that takes into account both the resolution of the spectrometer and the rounding of the sample,

$R_{M}\left(Q_{z}\right)=\frac{1}{\gamma \sqrt{2 \pi}} \int_{\infty}^{-\infty} d\left(\delta Q_{z}\right) R_{F}\left(Q_{z}+\delta Q_{z}\right) e^{-\left(\delta Q_{z}\right)^{2} / 2 \gamma^{2}}$

where for $Q>Q_{c}$

$$
R_{F}\left(Q_{z}\right) \approx \frac{\left(Q_{z}-Q_{z}^{\prime}\right)^{2}+\left(4 \pi / \lambda \mu Q_{z}^{\prime}\right)^{2}}{\left(Q_{z}+Q_{z}^{\prime}\right)^{2}+\left(4 \pi / \lambda \mu Q_{z}^{\prime}\right)^{2}}
$$

and

$$
Q_{z}^{\prime}=\left(\frac{Q_{z}^{2}-Q_{c}^{2}+\left[(2 / \mu)^{2}+\left(Q_{z}^{2}-Q_{c}^{2}\right)\right]^{1 / 2}}{2}\right)^{1 / 2}
$$

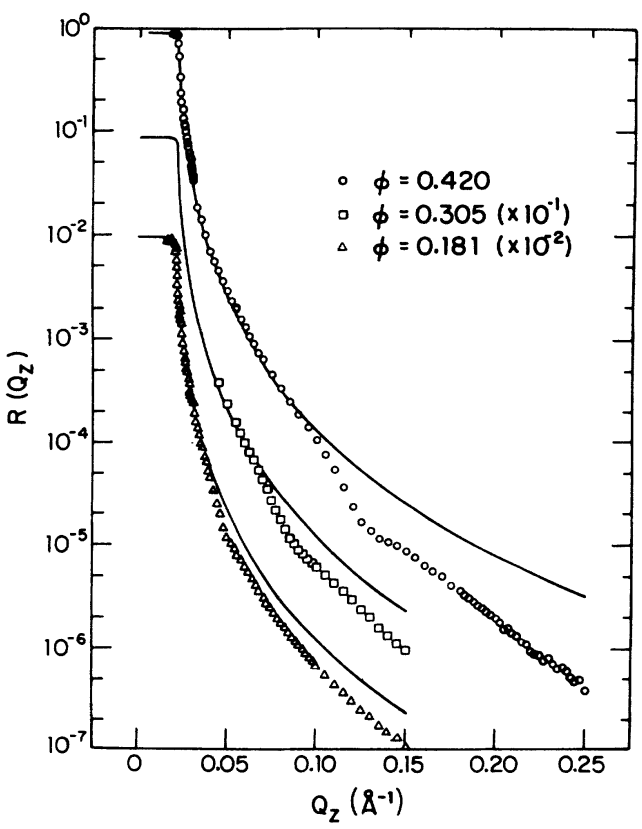

FIG. 2. Observed specular reflectivity intensity for samples with AOT volume fractions $\phi=0.420,0.305$, and 0.181 , normalized to the intensity of the direct beam. The solid lines represent the theoretical form of reflectivity $R_{F}\left(Q_{z}\right)$ expected for an abrupt step function interface with the bulk electron density of the respective material.

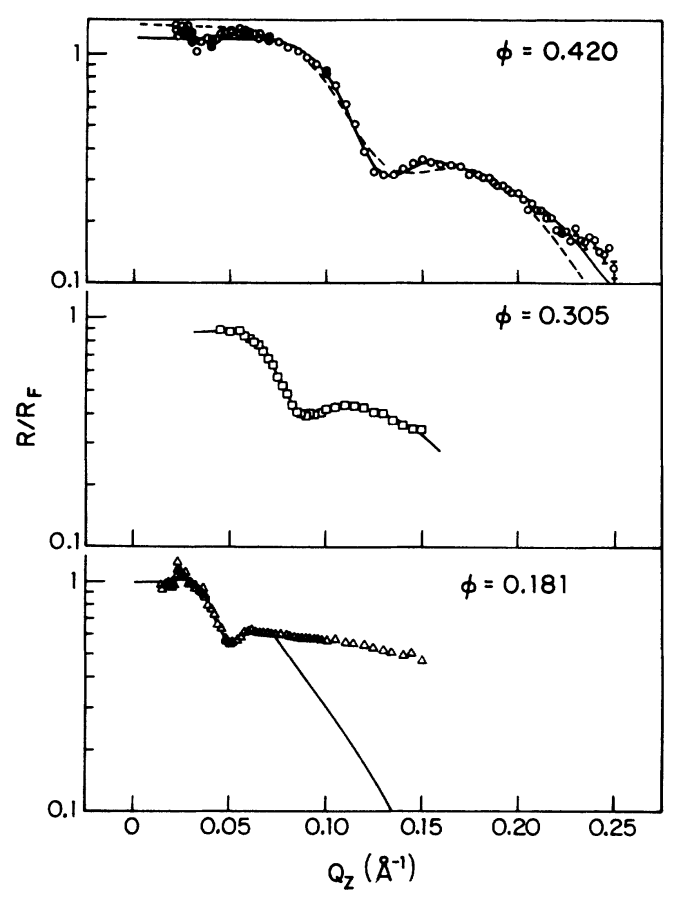

FIG. 3. The data points represent the data points from Fig. 2 divided by the respective Fresnel value: $\phi=0.420,0.305$, and 0.181 . The solid lines represent the best fits to a model of surface-induced layers as discussed in the text. For samples with $\phi=0.420$ and 0.305 the solid lines correspond to a model with two layers of micelles. The dashed line in the $\phi=0.420$ sample shows the inferior quality of the best fit for only one layer. The data for the sample with $\phi=0.181$ are qualitatively different from the others and have some features not fully explained by our model. The fit shown corresponds to one layer of micelles and was not substantially improved with two layers.

while for $Q<Q_{c}$

$$
R_{F}\left(Q_{z}\right)=1 \text {. }
$$

The convolution is only important for small values of $Q_{z}<0.03 \AA^{-1}$ where a nonlinear least-square fit obtains $\gamma \approx 8.0 \times 10^{-4} \AA^{-1}$. The critical $Q_{z}$ vectors and absorption lengths for the different samples are listed in Table I.

The solid lines through the data in Fig. 3 describe a theoretical model that will be discussed below. The most important qualitative information in these data is the prominent dip in the reflectivity at values of

$$
Q_{z}^{\prime(\text { surf })}=\left[\left(Q_{z}^{\text {(surf) }}\right)^{2}-Q_{c}^{2}\right]^{1 / 2}
$$

that scale with the volume fraction of AOT, $\phi$, in the same manner as the $Q_{0}(\phi)$ that characterizes the size of the micelles in the bulk. Table II lists the values of

TABLE I. Critical wave vectors and absorption lengths for the three concentrations studied.

\begin{tabular}{lcc}
\hline \hline$\phi$ & $Q_{c}\left(\AA^{-1}\right)$ & $\mu(\mathrm{cm})$ \\
\hline 0.420 & 0.02145 & 0.0976 \\
0.305 & 0.02110 & 0.1039 \\
0.181 & 0.02085 & 0.1229 \\
\hline \hline
\end{tabular}


TABLE II. Comparison between the positions for the minima in the reflectivity signal with the maxima in the diffuse scattering from the bulk: $Q_{z}^{\prime \text { (surf) }}$ is the scattering wave vector corresponding to the position of the minima in the specular reflection; $Q_{0}^{(\mathrm{fit})}$ corresponds to the measured radius of the ring of diffuse scattering from the material below the surface; and $Q_{0}(\phi)$ is the value expected for the stated volume fraction of AOT on the basis of the work of Kotlarchyk. The differences between $Q_{0}^{\text {(fit) }}$ and $Q_{0}(\phi)$ are probably due to small changes in concentration as discussed in the text. The diffuse scattering was not measured for the sample with $\phi=0.181$.

\begin{tabular}{ccccc}
\hline \hline$\phi$ & $\begin{array}{c}Q_{z}^{\prime \text { (surf) }} \\
\left(\AA^{-1}\right)\end{array}$ & $\begin{array}{c}Q_{0}^{\text {(fit) }} \\
\left(\AA^{-1}\right)\end{array}$ & $\begin{array}{c}Q_{0}(\phi) \\
\left(\AA^{-1}\right)\end{array}$ & $Q_{z}^{\prime \text { (surf) }} / Q_{0}(\phi)$ \\
\hline 0.420 & $0.130 \pm 0.005$ & $0.113 \pm 0.0015$ & 0.102 & $1.27 \pm 0.05$ \\
0.305 & $0.088 \pm 0.003$ & $0.073 \pm 0.002$ & 0.066 & $1.21 \pm 0.05$ \\
0.181 & $0.046 \pm 0.002$ & & 0.034 & $1.35 \pm 0.06$ \\
\hline \hline
\end{tabular}

$Q_{z}^{\prime(\text { surf) }}$ as well as the values for $Q_{0}^{(\text {fit })}$, as obtained below, from fitting the diffuse scattering from the bulk of the same samples. The model of the surface to be discussed below perdicts that the ratio of $Q_{z}^{\prime \text { (surf) }} / Q_{0}^{\text {(fit) }}=\left(\frac{3}{2}\right)^{1 / 2}$ which is very close to the measured ratio. Irregular variations in the data at the smallest wave vectors (i.e., at small $\theta$ ) are due a diverging projection of the beam height onto the finite length of the horizontal sample surface.

Transverse scans at several values of $Q_{z}$, as shown in Fig. 4 for the sample with $\phi=0.42$, cut through the sphere of diffuse scattering from the bulk. The solid lines through the diffuse scattering data in Fig. 4 are the results of nonlinear least-squares fits to the different data sets with the functional form

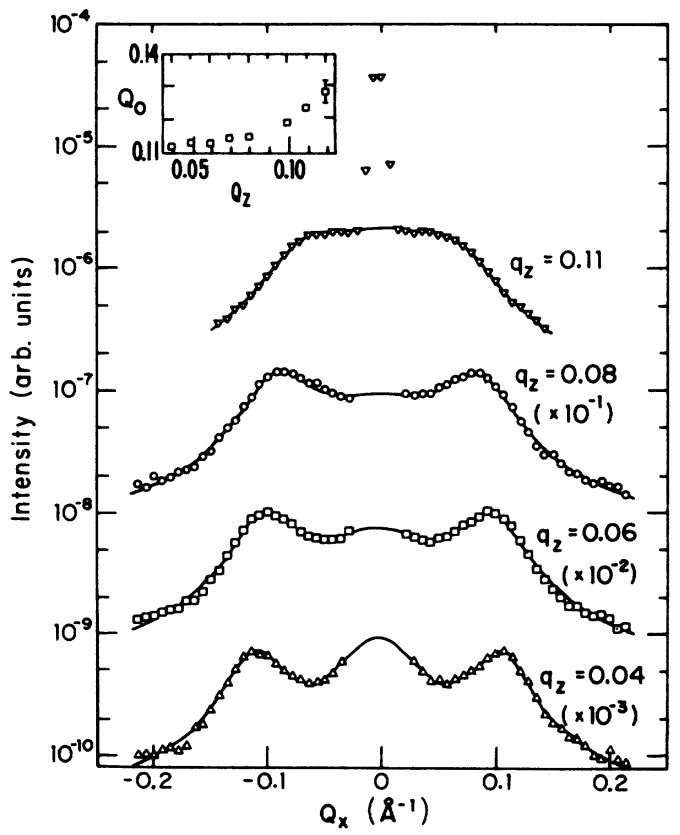

FIG. 4. Scans along $Q_{x}$ at different values of $Q_{z}$ to measure the diffuse ring of bulk scattering for the sample with $\phi=0.41$. The positions of the satellite peaks move in for larger values of $Q_{z}$ showing a constant $Q_{0}=\left(Q_{x}^{2}+Q_{z}^{2}\right)^{1 / 2}$. The inset displays the values of $Q_{0}$ obtained from the bulk data like those shown here. The variation in $Q_{0}$ at large $Q_{z}$ may be an artifact of the resolution of the spectrometer.

$$
S\left(Q_{x}\right)=A\left(\frac{1}{1+\xi^{2}\left(|Q|-Q_{0}\right)^{2}}\right)+\frac{B w^{2}}{Q_{x}^{2}+w^{2}}+\text { const , }
$$

where $|\mathbf{Q}|^{2}=Q_{x}^{2}+Q_{z}^{2}-Q_{c}^{2}$ and $Q_{0}$ is the radius of the sphere if refraction effects near to the critical angle are neglected. Note that $Q_{z}^{\prime}=\left(Q_{z}^{2}-Q_{c}^{2}\right)^{1 / 2}$ is the $z$ component of the wave vector inside the material. The second term is included to account for low-angle scattering near to the specular condition due to either diffuse surface scattering or from normal low-angle scattering from the bulk. The adjustable parameters were $A, B w \xi$, and $Q_{0}$. The inset in Fig. 4 displays the results obtained for $Q_{0}^{\text {(fit) }}$ from scans at different values of $Q_{z}$.

So long as $Q_{z} \ll Q_{0}$ the fitted results for $Q_{0}^{(\mathrm{fit})}$ are independent of $Q_{z}$ and yield the values displayed in Table II. The deviations for scans taken at values of $Q_{z} \approx Q_{0}$ suggest values of $Q_{0}^{\text {(fit) }}$ that are approximately $10 \%$ larger than the small $Q_{z}$ values. Although it is possible that the sample below the surface is not truly an isotropic powder and that the diffuse scattering ring is not cylindrical, we do not believe the present data set warrant such a conclusion. Other artifacts associated with the spectrometer resolution function, or other small angle scattering effects could also give rise to the observed effect. Further measurements should be undertaken to obtain data that would justify a more detailed line-shape analysis; however, for the present, the only parameter that we want to extract from the data for the scattering from the bulk is the value of $Q_{0}^{(\mathrm{fit})}$ for the samples that were studied.

This is particularly important, in view of the fact that for the same values of the volume fraction of $\phi$, the neutron diffraction results obtained values of $Q_{0}$ that were systematically smaller than those observed here. In view of the fact that our sample chambers has a free volume above the liquid that is approximately $10^{2}$ the volume of the sample, it is certain that we have had some evaporation of both $\mathrm{D}_{2} \mathrm{O}$ and decane. In any event, since the bulk and surface properties were measured in situ on the same sample, the discrepancy should not affect the conclusions to be drawn below.

In order to develop some understanding of the implications that can be drawn from the reflectivity data (illustrated in Figs. 2 and 3 ) on the electron density variations near to the surface, we used the following empirical model for $\langle\rho(z)\rangle$. This includes the density change at the 
air/liquid interface and up to two surface layers of micelles:

$$
\begin{aligned}
\langle\rho(z)\rangle= & \left(\rho_{\text {bulk }} / 2\right)\left[\operatorname{erf}\left(z / \sigma_{1}\right)+1\right] \\
& +\sum_{n=1}^{2} A_{n} f\left(z-z_{0}-(n-1) D_{0}\right),
\end{aligned}
$$

where

$$
f\left(s_{n}\right)=\left(1-\frac{s_{n}^{2}}{\sigma_{2}^{2}}\right) \exp \left(-s_{n}^{2} / 2 \sigma_{2}^{2}\right) .
$$

Since the error function varies from -1 to 1 over a range of the order of $\Delta z \sim 2 \sigma_{1}$ the leading term is a convenient form for representing the variation in the electron density between the vapor and the bulk. The remaining terms are just one of a number of equivalent empirical forms that might have been used to construct a function representing structure normal to, and localized near, the surface. ${ }^{17}$ This particular form is convenient since it has zero average and the Fourier transformations of the Gaussian integrals can be done analytically. We will see below how this function can be related to a more physical model. However, since the theoretical form for the reflectivity is obtained from the Fourier transform, and since the reflectivity is only measured over a limited range of $Q_{z}$, the only parts of the model that have physical significance are the lowest Fourier components.

In view of the fact that the first term already describes the average density far below the surface, the second term must average to zero. Aside from a constant of normalization, a suitable function $f\left(s_{n}\right)$ for modeling the average form factor of the surface induced layers is the second derivative of a Gaussian: $A_{n}$ is the amplitude associated with the $n$th layer, $D_{0}$ is the spacing between successive layers, $z_{0}$ locates the position of the first layer relative to the surface, and $\sigma_{2}$ is a parameter that describes the shape. In systems such as the nematic phase of certain thermotropic liquid crystals the number of layers $N$ is large, and the Fourier transform of the derivative of this density would be a function whose real part is sharply peaked $Q_{z} \approx\left(2 \pi / D_{0}\right)$ and with an imaginary part that has a dispersive shape. ${ }^{13,14}$ In fact, the best fits to the present data for samples (a) and (b) obtain values of $N \approx 2$ with $A_{2} \approx 0.2 A_{1}$. For sample (c) the fit for $N \approx 2$ was not substantially better than for $N \approx 1$. The position and shape of the minimum depend primarily on $z_{0}, D_{0}$, and $\sigma_{2}$. The remaining parameter $\sigma_{1}$, which describes the width of the transition region between the vapor and the bulk, relates directly to the way the average reflectivity falls off with increasing $Q_{z}$. Since this is primarily related to the sharpness of the interface with the vapor it is not particularly important for understanding the oscillatory structure in the electron density below the surface.

The solid lines in Fig. 3 demonstrate the best fits that we have been able to obtain for this parametrization with parameters that are listed in Table II. The dashed line for sample (a) in Fig. 3 shows the inferior fit for $N \approx 1$.

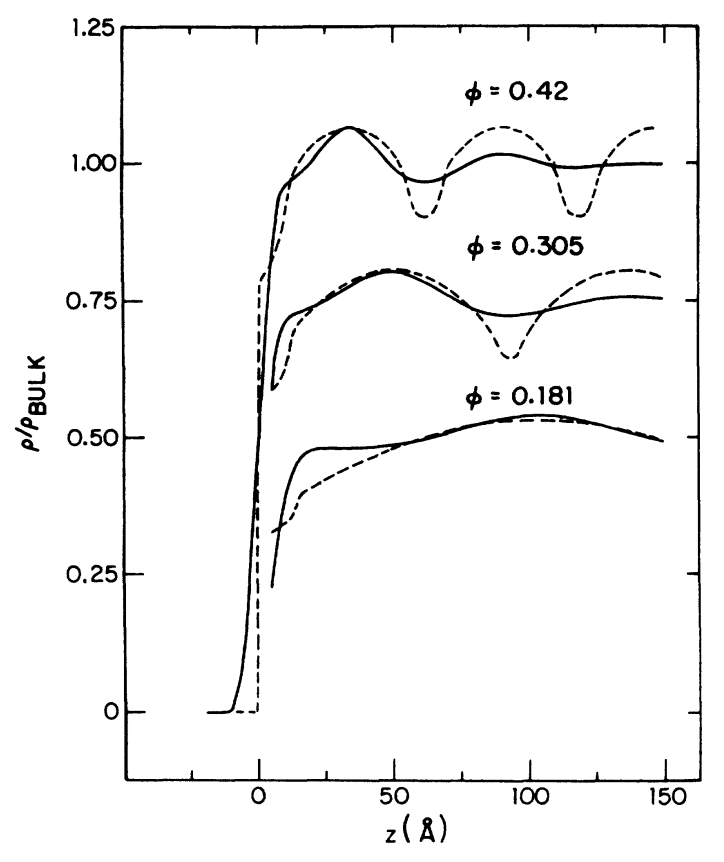

FIG. 5. The solid lines are the surface densities that represent the best fit of the model discussed in the text to the reflectivity data: $\phi=0.420,0.305$, and 0.181 . The dashed lines represent a simple model in terms of spherical micelles at the surface.

The solid lines in Fig. 5 show the real space density corresponding to these fits. The physical significance of this parametrization of the surface density can be related to the simple droplet model, shown in Fig. 6, in which monodisperse micelles are aligned by the free surface. Although a full theoretical treatment of the surface structure would allow for both polydispersivity and positional disorder, neither of these details have a major effect on the structure nearest to the surface. In this model micelles, separated by a distance $d$, are described as having an inner water core of radius $R_{c}$, surrounded by a shell of AOT molecules, with an outer radius $R_{m} \cdot{ }^{10}$ The micelles are in a bath of decane and since there is a thin layer of decane separating adjacent droplets the nearest-neighbor distance $d$ is slightly large than $2 R_{m}$. The values of $R_{c}$, $R_{m}$, and $d$ are calculated from volumetric considerations that are illustrated by consideration of Fig. 6 . The inner region of the droplet is filled with $\mathrm{D}_{2} \mathrm{O}$ and coated with a layer $\sim 5 \AA$ thick corresponding to the head group of the AOT molecule. The outside radius of the head group region, which is the same as the inner radius of the outer layer, is $R_{c}$. The outer radius of the droplet is $R_{m}$ and the $\sim 10-\AA$ region between $R_{m}$ and $R_{c}$ is filled with the hydrocarbon tail of the AOT molecule. All space not occupied by the micelles is filled with decane. Figure 6(b) illustrates the electron density as a function of $r$ for a hypothetical isolated droplet in decane. The model illustrated in Fig. 6(a) consists of two layers of micelles, or droplets at the free surface. We will show below that this model does account for the structure nearest to the sur- 
face. A more complete theory would be necessary to account for the loss of positional order with increasing distance from the surface; however, this model is adequate for the structure in the immediate vicinity of the surface.

Following Kotlarchyk et al. ${ }^{10}$ we calculate the dimensions of the micelle from volumetric considerations by assuming an fcc lattice. For the samples with $\phi=0.420$ and 0.305 the value of $d$ was obtained from the diffuse scattering measurements $d=\pi \sqrt{6} / Q_{0}$ (extraneous small angle scattering precluded suitable diffuse scattering measurements for the sample with $\phi=0.181$ ). The radii $R_{c}$ and $R_{m}$ are then computed from the following expressions:

$$
\begin{aligned}
& R_{c}=\alpha(1-\phi)^{1 / 3} d \text { where } \alpha=0.6964, \\
& R_{m}=\left(\frac{1+\phi}{1-\phi}\right)^{1 / 3} R_{c} .
\end{aligned}
$$

The results listed in Table III would differ by only $3 \%$ if a bcc lattice was used. The value for $R_{m}-R_{c} \approx 9 \AA$ is approximately equal to the length of the AOT hydrocarbon chain.

The electron density for the head group region was calculated by taking the polar head group of the surfactant molecule to be approximately $5 \AA$ thick and with an area of the order of $51 \AA^{2}$ for a total volume of $225 \AA^{3}$. Counting up the number of electrons in the polar head this obtains an electron density of $\rho_{\text {head }}=4.89 \times 10^{23}$ $e / \mathrm{cm}^{3}$ for the head region. When the above volume for the head is subtracted from the volume of the molecule as a whole $\left(612 \AA^{3}\right)$ one obtains a volume for the tail of 387 $\AA^{3}$ that leads to an electron density for the tail region of $\rho_{\text {tail }}=3.36 \times 10^{23} \mathrm{e} / \mathrm{cm}^{3}$. The density of $\mathrm{D}_{2} \mathrm{O}$ is 1.104 $\mathrm{g} / \mathrm{cm}^{3}$ corresponding to $3.33 \times 10^{23} \mathrm{e} / \mathrm{cm}^{3}$ for the inner region of the droplet and that of decane is $0.730 \mathrm{~g} / \mathrm{cm}^{3}$ corresponding to $2.54 \times 10^{23} \mathrm{e} / \mathrm{cm}^{3}$ in the space between the micelles.

The model surface electron density is constructed by assuming that at the surface there is a monolayer of micelles, and that successive layers are formed by close packing of further micelles. For simplicity we take the surface to be the (111) surface of a fcc lattice with one micelle per unit cell. We do not attempt to include effects of polydispersivity. The average electron density as a func- tion of distance from the surface is then calculated from the above model with no further adjustable parameters.

A qualitative description of the model is that right at the surface the electron density has the small value of pure decane $\left(2.5 \times 10^{23} e^{-} / \mathrm{cm}^{3}\right)$. The thickness of this layer is vanishingly small since the droplets come up to and touch the surface. Continuing into the solution the decane is increasingly replaced by the droplets and, due to the higher density of water at the core, the density rises to a maximum at a depth approximately equal to $R_{m}$ that corresponds to the center of the first layer of micelles. After the maximum the density decreases until the micelles of the next layer appear. Without including a specific mechanism for loss of order, the density oscillates with a period $D_{0}=d \sqrt{2 / 3} \sim 2 R_{m} \sqrt{2 / 3}$ that is related to the nearest-neighbor distance $d$ of the micelles packed in the geometry of the fcc lattice. The dashed line in Fig. 5 illustrates the result for this model. The structure nearest to the surface, as indicated by both the position and amplitude of the first maxima is essentially identical for both the $a d h o c$ form and for the micellar model (e.g., $z_{0} \approx R_{m}$ and $A_{1}$ agree well with the droplet model). In principle, polydispersivity in the micelle size would produce decaying amplitudes for the micellar model; however, in order to produce a decay as rapid as that of the ad hoc model, the polydispersivity would have to allow for size variations of the order of a factor of 2 .

It is also possible that the micelles are not strictly spherical and that the subsequent maxima and minima are attenuated due to some combination of fluctuations in the droplet shape combined with orientational disorder. ${ }^{18}$ Another possibility that would also be consistent with the measured reflectivity would be if there were a high density of defects in the triangular packing of the first micellar layer. This could have the effect of producing a sizeable variation in the position of the second layer and if there are similar defects in subsequent layers this would also reduce the amplitude as observed. In particular, for the sample with volume fraction $\phi=0.181$, the mixture is near to the phase separation boundary and it is possible that surface effects might stabilize smaller droplets that could pack in the interstices between the larger droplets. This would also have the effect reducing the disparity between the solid and dashed lines in the region near to the surface in Fig. 5 by enhancing the polydispersivity that would wash out the subsequent maxima and minima in the electron density away from the surface.

TABLE III. Details of models for the AOT surface. The quantities $2 \pi / Q_{0}, z_{0}, A_{1}, Z_{2}, \sigma_{1}, \sigma_{2}$ are the results of the fitting of the ad hoc model to the specular reflectivity data. The quantities $d, R_{c}, R_{m}$ are calculated from volumetric considerations for a droplet mod-

\begin{tabular}{|c|c|c|c|c|c|c|c|c|c|c|}
\hline \multirow[b]{2}{*}{$\phi$} & \multicolumn{4}{|c|}{ Ad hoc model } & \multirow[b]{2}{*}{$\begin{array}{c}\sigma_{2} \\
(\AA)\end{array}$} & \multirow[b]{2}{*}{$\begin{array}{l}\sigma_{1} \\
(\AA)\end{array}$} & \multicolumn{4}{|c|}{ Droplet model } \\
\hline & $\begin{array}{l}D_{0} \\
(\AA)\end{array}$ & $\begin{array}{c}z_{0} \\
(\AA) \\
\end{array}$ & $A_{1}$ & $A_{2}$ & & & $\begin{array}{c}d \\
(\stackrel{\AA}{\mathrm{A}}) \\
\end{array}$ & $\begin{array}{l}R_{c} \\
(\AA)\end{array}$ & $\begin{array}{l}R_{m} \\
(\AA)\end{array}$ & $\begin{array}{c}(\sqrt{2 / 3}) d \\
(\AA)\end{array}$ \\
\hline 0.420 & $56 \pm 1$ & $34.3 \pm 0.3$ & $0.066 \pm 0.022$ & $0.018 \pm 0.002$ & 15 & $6.3 \pm 0.1$ & 69 & 25 & 34 & $56 \pm 1$ \\
\hline 0.305 & $83 \pm 2$ & $49.5 \pm 0.4$ & $0.053 \pm 0.001$ & $0.009 \pm 0.001$ & 25 & $6.5 \pm 0.08$ & 106 & 41 & 50 & $86 \pm 2$ \\
\hline 0.181 & & $104 \pm 1$ & $0.041 \pm 0.002$ & & 40 & $11 \pm 0.2$ & $226^{a}$ & $93^{a}$ & $104^{\mathrm{a}}$ & $185^{\mathrm{a}}$ \\
\hline
\end{tabular}
el starting from the value of $d=\pi \sqrt{6} / Q_{0}^{(\text {fit })}$ obtained from the diffuse scattering.

${ }^{\mathrm{a}}$ Taken from surface data. 
(a)

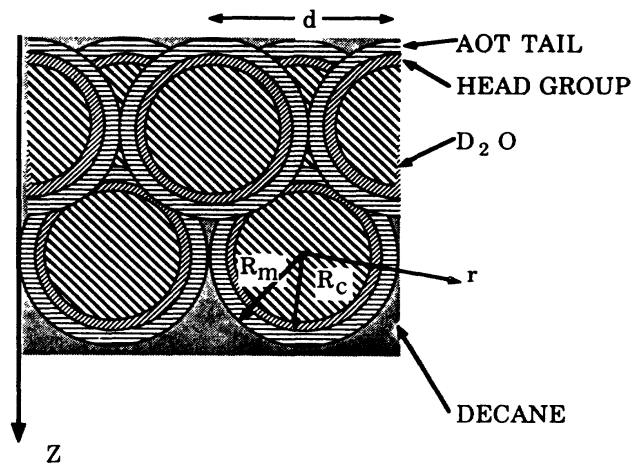

(b)

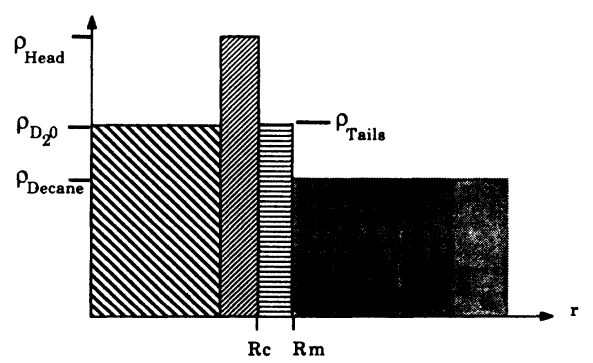

FIG. 6. Schematic illustration of the droplet model discussed in the text. The micelle, or droplet, is represented as concentric spherical shells. The inner sphere is filled with only water (or $\mathrm{D}_{2} \mathrm{O}$ in the present samples). The water sphere is surrounded by a shell approximately $5 \AA$ thick corresponding to the region occupied by the head group of the AOT molecule. The outer shell, approximately $9 \AA$ thick is occupied by the tails of the AOT molecule. The outside radius of the head group region, or the inner radius of the tail region, is labeled $R_{c}$. Since the entire droplet is coated by a thin layer of decane that is not explicitly shown, the nearest-neighbor distance $d / 2$ is slightly larger than $R_{m}$. (a) Illustration of the packing of droplets at the surface. (b) Electron density, as a function of radius for a hypothetical individual droplet in decane.

\section{DISCUSSION}

Assuming the correctness of the Kotlarchyk model for the bulk as close packed spherical micelles, it is difficult to imagine a structure other than one with these same micelles at the surface that could explain the destructive interference displayed by the reflectivity. The general agreement between the ad hoc model and the spherical micelle model for the near surface structure is rather persuasive on this point. On the other hand, there are a number of open questions that should be investigated by further experiments.

Firstly, these measurements should be repeated under conditions where the sample concentration can be more closely controlled. As we noted above the values of $Q_{0}$ obtained from the diffuse scattering from the bulk of the samples were systematically larger than the results obtained by Kotlarchyk et al. using neutron diffraction. Although this should not affect our result that micelles extend up to the surface, it would be interesting to see if there is any evidence for a systematic variation in micelle size with distance from the surface. This might be studied, for example, by measuring $Q_{0}$ with $x$ rays of varying wavelength that penetrate into the bulk different amounts for the same $Q_{z}=(4 \pi / \lambda) \sin (\theta){ }^{16}$

Secondly, the angular dependence of the reflectivity of the sample with $\phi=0.181$ deviates significantly from the model at $Q_{z} \geq 1.5 Q_{0}$. Measurements on other concentrations should be extended to larger angles to see if this is a more general feature of the AOT microemulsion surface. In particular the relatively constant value of $R\left(Q_{z}\right) / R_{F}\left(Q_{z}\right) \approx 0.6$ out to values of $Q_{z} \approx 0.15 \AA^{-1}$ suggests that for this sample the surface structure should include a relatively sharp step in the electron density. It is not clear how this would relate to the model mentioned above, and without further measurements, or a sound theoretical suggestion, further speculation would be idle.

Thirdly, the angular dependence of the diffuse scattering from the bulk should be studied in more detail. The origin of the small angle scattering off of the specular condition would be particularly interesting. As we mentioned above, one microscopic model that might be able to explain the relatively broad width of the destructive minima in the reflectivity (e.g., the fact that the real space density of the ad hoc model only includes two terms) might be a high density of defects in the way micelles are packed within the surface layer. This should manifest itself in diffuse scattering.

Finally, it would be interesting to study this, and similar systems, as a function of temperature, and also at a greater variety of compositions in order to determine how the surface changes near bulk transitions.

In summary we find that the angular dependence of $\mathrm{x}$ ray specular reflectivity from the AOT microemulsion surface is consistent with two layers of micelles with sizes and lattice spacings that agree with the sizes of micelles in the bulk.

\section{ACKNOWLEDGMENTS}

The research reported here was supported by the U.S. National Science Foundation through Grant No. DMR85-13523 and through the Harvard Materials Research Laboratory, Grant No. DMR-86-14003.

\footnotetext{
*Present address: Laboratoire de Physique des Solides, Bâtiment 510, Université Paris-Sud, 91405 Orsay Cédex, France.

†Present address: Department of Physics, Brookhaven National Laboratory, Upton, NY 11973.

${ }^{1}$ See, for example, Physics of Complex and Supermolecular Fluids, edited by S. A. Safran and N. A. Clark (Wiley, New York, 1987).
}

${ }^{2}$ P. Ekwall, L. Mandell, and K. Fontell, J. Colloid Inter. Sci. 33, 215 (1970).

${ }^{3}$ G. L. Kirk, S. M. Gruner, and D. L. Stein, Biochem. 23, 1093 (1984).

${ }^{4}$ S. A. Safran and L. A. Turkevich, Phys. Rev. Lett. 50, 1930 (1983).

${ }^{5}$ R. A. Day, B. M. Robinson, J. M. R. Clarke, and J. V. Doher- 
ty, J. Chem. Soc. Faraday Trans. I. 75, 132 (1979).

${ }^{6}$ M. Zulauf and H. F. Elcke, J. Phys. Chem. 83, 480 (1979).

${ }^{7}$ P. C. Cabos and P. Delord, J. Appl. Cryst. 12, 502 (1979).

${ }^{8}$ T. Assih, F. Larche, and P. Delord, J. Colloid Inter. Sci. 89, 35 (1982).

${ }^{9}$ M. Kotlarchyk, S. H. Chen, J. S. Huang, and M. W. Kim, Phys. Rev. A 29, 2054 (1984).

${ }^{10}$ M. Kotlarchyk, S. H. Chen, J. S. Huang, and M. W. Kim, Phys. Rev. Lett. 53, 941 (1984).

${ }^{11}$ J. S. Huang and M. Kotlarchyk, Phys. Rev. Lett. 57, 2587 (1986).

${ }^{12}$ B. M. Ocko, A. Braslau, P. S. Pershan, J. Als-Nielsen, and M. Deutsch, Phys. Rev. Lett. 57, 94 (1986).
${ }^{13}$ P. S. Pershan, A. Braslau, A. H. Weiss, and J. Als-Nielsen, Phys. Rev. A 35, 4800 (1987).

${ }^{14}$ P. S. Pershan and J. Als-Nielsen, Phys. Rev. Lett. 52, 759 (1984).

${ }^{15}$ A. Braslau, M. Deutsch, P. S. Pershan, A. H. Weiss, and J. Als-Nielsen, Phys. Rev. Lett. 54, 114 (1985).

${ }^{16} \mathrm{~J}$. Als-Nielsen and P. S. Pershan, Nucl. Instrum. Methods 208, 545 (1983).

${ }^{17}$ For example, we have also used an exponentially decaying sine wave and obtained results that are essentially identical to those presented below.

${ }^{18}$ J. S. Huang, S. T. Milner, B. Farago, and D. Richter, Phys. Rev. Lett. 59, 2600 (1987). 


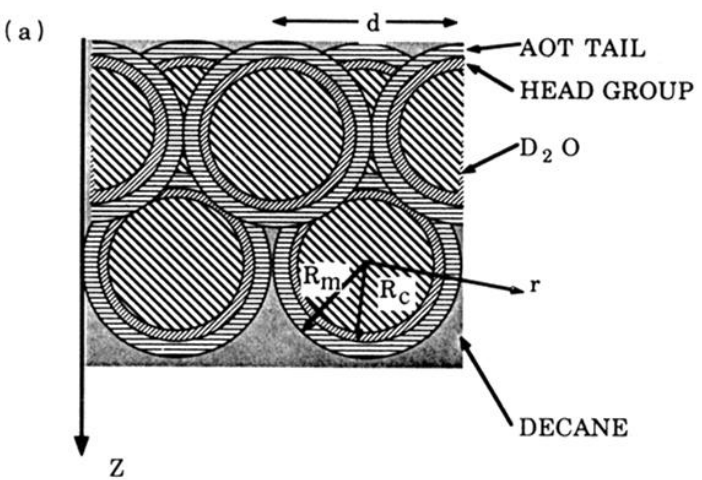

(b)

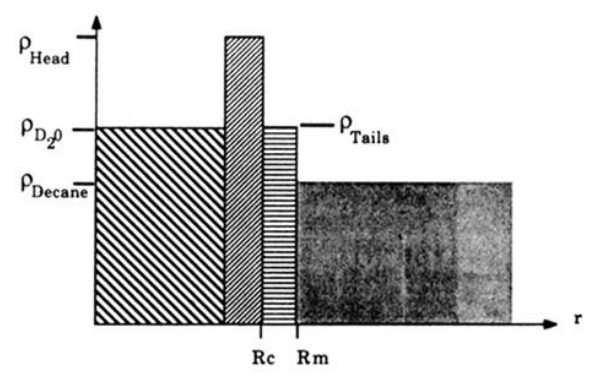

FIG. 6. Schematic illustration of the droplet model discussed in the text. The micelle, or droplet, is represented as concentric spherical shells. The inner sphere is filled with only water (or $\mathrm{D}_{2} \mathrm{O}$ in the present samples). The water sphere is surrounded by a shell approximately $5 \AA$ thick corresponding to the region occupied by the head group of the AOT molecule. The outer shell, approximately $9 \AA$ thick is occupied by the tails of the AOT molecule. The outside radius of the head group region, or the inner radius of the tail region, is labeled $R_{c}$. Since the entire droplet is coated by a thin layer of decane that is not explicitly shown, the nearest-neighbor distance $d / 2$ is slightly larger than $R_{m}$. (a) Illustration of the packing of droplets at the surface. (b) Electron density, as a function of radius for a hypothetical individual droplet in decane. 